\title{
Estudo de heterogeneidade de variâncias na avaliação genética de bovinos de corte da raça Nelore
}

\author{
Cláudio Vieira de Araújo ${ }^{1}$, Thereza Cristina Borio dos Santos Calmon de Bittencourt ${ }^{2}$, \\ Simone Inoe Araújo ${ }^{1}$, Raysildo Barbosa Lôbo ${ }^{3}$, Luiz Antônio Framartino Bezerra ${ }^{3}$
}

\footnotetext{
1 Instituto de Ciências Agrárias e Ambientais - Universidade Federal de Mato Grosso - Campus de Sinop - 78550-000 - Sinop, MT

2 Departamento Produção Animal - Escola de Medicina Veterinária - Universidade Federal da Bahia - 40000-000 - Salvador, BA.

${ }^{3}$ ANCP- Associação Nacional de Criadores e Pesquisadores - Ribeirão Preto, SP.
}

RESUMO - Verificou-se a influência da heterogeneidade de variâncias na avaliação genética de bovinos de corte da raça Nelore. Dados de pesos padronizados aos 365, 450 e 550 dias de idade foram estratificados com base no desvio-padrão fenotípico dos rebanhos para cada peso. Nas análises de múltiplas características, em que o mesmo peso foi considerado característica distinta em cada classe de desvio-padrão, as variâncias genéticas e residuais aumentaram com o desvio-padrão da classe. As estimativas de herdabilidade foram iguais a 0,34 e 0,36; 0,41 e 0,41, 38 e 49 nas classes de alto e baixo desvio-padrão fenotípico para os pesos aos 365, 450 e 550 dias, respectivamente. As correlações genéticas entre o mesmo peso nas classes de baixo e alto desvio-padrão foram iguais a 0,71; 0,80; e 0,84 para os pesos aos 365, 450 e 550 dias, respectivamente. As correlações de Spearman entre os valores genéticos, obtidos de análises múltiplas e de análise geral (sem as classes), e entre o mesmo peso nas classes de desvios-padrão, reduziram à medida que aumentou a intensidade de seleção sobre os reprodutores. A presença de heterogeneidade de variâncias causa maior impacto sobre a avaliação genética dos reprodutores sob intensidade de seleção elevada, sendo interessante sua consideração no processo de avaliação genética.

Palavras-chave: avaliação genética, bovinos de corte, interação genótipo × ambiente, parâmetros genéticos, seleção

\section{Study of heterogeneity of variances on genetic evaluation of Nellore beef cattle}

\begin{abstract}
Data from Nellore beef cattle breed were used to study the influence of variance heterogeneity on genetic evaluation. Adjusted weights at 365, 450 and 550 days of age were classified as high and low classes of standard deviation, based on phenotypic standard deviation of herds in each weight. At multiple trait analyses, where the same weight was considered a distinct characteristic regarding each class of standard deviation, genetic and residual variances increased as the standard deviation of the class increased. Genetic correlations between the same weight, in high and low phenotypic standard deviation classes were $0.71,0.80$ and 0.84 for weights at 365, 450 and 550 days, respectively. Spearman correlations between breeding values obtained from multiple trait analyses and general analysis (without classes), and between classes of standard deviations, lowered as the intensity of selection on sires increased. The presence of heterogeneity of variances has greater impact on the genetic evaluation of breeding under high intensity of selection, it is interesting your consideration in the process of genetic evaluation.
\end{abstract}

Key Words: beef cattle, genetic evaluation, genetic parameters, genotype $\times$ environment interaction, selection

\section{Introdução}

O maior interesse de quem necessita utilizar reprodutores e matrizes é que esses animais sejam avaliados e ordenados corretamente, independentemente do processo de avaliação e, ou, do local de criação. A avaliação genética pode ser influenciada pela precisão com que os parâmetros genéticos e ambientais utilizados são estimados, que pode ser afetada por vários fatores, como a interação genótipo $\times$ ambiente, conceituada como diferenças nas respostas dos genótipos em diferentes ambientes, que, quando presente, pode levar a viés no processo de avaliação genética, ocasionando alteração no ordenamento dos genótipos realizado com base no desempenho em diferentes ambientes, ou seja, a prole pode não repetir o desempenho do progenitor, caso seja criada em país, estado, microrregião ou mesmo rebanho diferentes daqueles em que seus pais foram criados.

A heterogeneidade de variância pode ser vista como uma forma primária de interação genótipo $\times$ ambiente $\mathrm{e}$ pode comprometer o resultado do processo de seleção caso a fonte dessa heterogeneidade sejam fatores ambientais. Consequentemente, a seleção será realizada mais em função do ambiente que propriamente pelo genótipo do animal. 
A esse respeito, mesmo os procedimentos que produzem avaliação genética com propriedades de melhor predição linear não-viesada (BLUP) para as soluções, estão sujeitos a erros e, consequentemente, pode comprometer o progresso genético esperado pela seleção, quando realizada entre rebanhos.

Revisando esse tema, Martins (2002) destacou como causas da heterogeneidade de variâncias entre rebanhos a composição racial ou as diferenças na composição genética da população, causadas por diferenças na intensidade de seleção, além da interação genótipo $\times$ ambiente.

No âmbito da avaliação genética de animais de reprodução sujeita à influência da interação genótipo $\times$ ambiente, esse assunto tem sido abordado de forma simples. Campelo et al. (2003) estudaram a influência da heterogeneidade de variâncias na avaliação genética de bovinos de corte da raça Tabapuã utilizando pesos padronizados aos 120, 240 e 420 dias de idade e estratificando os rebanhos em três classes de desviopadrão. Verificaram maiores estimativas de variâncias genéticas e residuais na classe de maior desvio-padrão, além de correlações genéticas inferiores a 0,80 entre o mesmo peso tratado como característica diferente nas classes de baixo e alto desvio-padrão, porém, concluíram que os reprodutores seriam classificados de forma similar, independentemente se considerada a presença de variâncias heterogêneas nas análises.

Por outro lado, Carneiro et al. (2006), em estudo com simulação de dados, observaram que a presença de médias genéticas diferentes entre rebanhos afeta a acurácia da predição dos valores genéticos de touros, principalmente de vacas e touros jovens, acarretando erros na classificação dos animais geneticamente superiores.

Com este estudo objetivou-se avaliar a influência de variâncias heterogêneas nos dados sobre as estimativas de parâmetros genéticos de características de crescimento em bovinos de corte da raça Nelore e seu impacto na avaliação genética de reprodutores, considerando-se também a intensidade de seleção.

\section{Material e Métodos}

Nesse estudo foram utilizadas informações de 12.127, 12.122 e 12.117 registros de pesos padronizados aos 365 , 450 e 550 dias de idade, respectivamente, provenientes de animais da raça Nelore, proles de 211 reprodutores criadas em 34 rebanhos localizados nos estados de Goiás, Mato Grosso do Sul, Minas Gerais e São Paulo, participantes do
Programa de Melhoramento Genético da Raça Nelore Nelore Brasil da Associação Nacional de Criadores e Pesquisadores, para avaliação da influência de variâncias heterogêneas nos dados sobre a classificação genética dos animais.

Nas análises estatísticas, foram utilizados grupos de contemporâneos contemplando efeitos fixos de rebanho, ano e estação de nascimento dos animais e sexo do animal. Os meses de nascimento foram agrupados em estações 1 e 2, que correspondem aos meses de menor e maior incidência de chuvas (de abril a setembro e de outubro a março), respectivamente. A idade da vaca ao parto oscilou de 22 a 160 meses, com média de 67,10 meses.

A matriz de numeradores dos coeficientes de parentesco (NRM) utilizada nas análises foi obtida de arquivo com pedigree contendo 53.763 animais, dos quais 2.684 apresentaram grau de endogamia, o que levou a um coeficiente médio de endogamia de 0,047.

Para avaliar a influência de variâncias heterogêneas nos dados sobre a classificação genética dos animais, foram realizadas análises estatísticas, para cada peso utilizando-se o banco de dados completo, e análises com os dados de cada peso agrupados em duas classes. Utilizou-se o valor da mediana da distribuição dos desviospadrão fenotípico dos rebanhos para cada peso padronizado como critério para a estratificação dos rebanhos em duas classes de desvio-padrão fenotípico (alto e baixo), sendo, respectivamente, os valores de 30,0; 36,1 e 43,0 para os pesos padronizados aos 365, 450 e 550 dias de idade.

O modelo linear misto utilizado para a obtenção de componente de variância para os efeitos genético aditivo, de ambiente permanente e ambiente temporário e, posteriormente, para predição de valores genéticos, considerando os pesos padronizados aos 365, 450 e 550 dias em análise geral, ou seja, que desconsidera as diferenças entre as variâncias das classes de desvios-padrão fenotípico, é representado como

$y=X \beta+Z_{1} a+Z_{2} p+e$

em que: $y=$ vetor de observações referente ao peso padronizado; $X=$ matriz de incidência de níveis dos efeitos fixos; $\beta$ = vetor de efeitos fixos referentes às classes: grupo de contemporâneos e efeito linear e quadrático da idade da vaca ao parto, como covariáveis; $\mathrm{Z}_{1}=$ matriz de incidência dos valores genéticos; $a$ = vetor de valores genéticos dos animais; $Z_{2}=$ matriz de incidência dos efeitos de ambiente permanente materno; $p=$ vetor de valores do efeito de ambiente permanente materno; $e=$ vetor de resíduos da mesma dimensão de $y$. 
As pressuposições acerca da distribuição dos vetores $y, a$ e $e$ são descritas como

$$
\left[\begin{array}{c}
y \\
a \\
p \\
e
\end{array}\right] \sim\left\{\left[\begin{array}{c}
X \beta \\
\phi \\
\phi \\
\phi
\end{array}\right],\left[\begin{array}{cccc}
Z_{1} G Z_{1}^{\prime}+Z_{2} P Z_{2}^{\prime}+R & Z G & Z P & R \\
G Z^{\prime} & G & \phi & \phi \\
P Z^{\prime} & \phi & P & \phi \\
R & \phi & \phi & R
\end{array}\right]\right\},
$$

em que: $G=A \sigma_{a}^{2} ; P=I_{n} \sigma_{P}^{2}$ e $R=I_{n} \sigma_{e}^{2}$, em que $A=$ matriz de parentesco entre os indivíduos, de ordem igual ao número de indivíduos $(\mathrm{N}) ; \sigma_{a}^{2}=$ variância genética aditiva para o peso padronizado; $\sigma_{P}^{2}=$ variância de ambiente permanente materno; $\sigma_{e}^{2}=$ variância residual; e $\mathrm{I}_{n}=$ matriz identidade de ordem $n$, em que $n$ é o número total de observações .

Para as análises de cada peso padronizado, considerado uma característica distinta em cada classe de desvio-padrão, levando-se em conta a distribuição conjunta das características, empregou-se o seguinte modelo

$$
y=X \beta+Z_{1} a+Z_{2} p+e,
$$

em que

$y=\left[\begin{array}{l}y_{1} \\ y_{2}\end{array}\right], X=\left[\begin{array}{cc}X_{1} & 0 \\ 0 & X_{2}\end{array}\right], \beta=\left[\begin{array}{l}\beta_{1} \\ \beta_{2}\end{array}\right], Z_{1}=\left[\begin{array}{cc}Z_{11} & 0 \\ 0 & Z_{12}\end{array}\right], a=\left[\begin{array}{l}a_{1} \\ a_{2}\end{array}\right]$, $Z_{2}=\left[\begin{array}{cc}Z_{21} & 0 \\ 0 & Z_{22}\end{array}\right], p=\left[\begin{array}{l}p_{1} \\ p_{2}\end{array}\right], e=\left[\begin{array}{l}e_{1} \\ e_{2}\end{array}\right]$.

em que: $y_{1}$ e $y_{2}=$ pesos padronizados nas classes de baixo e alto desvios-padrão fenotípico, respectivamente; $X_{\mathrm{i}}=$ matriz de incidência de níveis dos efeitos fixos na classe de desvio-padrão i; $\beta_{\mathrm{i}}=$ vetor de efeitos fixos na classe de desvio-padrão i; $Z_{1 i}=$ matriz diagonal de incidência dos valores genéticos na classe de desvio-padrão i; $a_{\mathrm{i}}=$ vetor de valores genéticos de cada animal na classe de desviopadrão i; $Z_{2 i}=$ matriz diagonal de incidência dos valores efeito de ambiente permanente materno na classe de desvio-padrão $\mathrm{i} ; p_{\mathrm{i}}=$ vetor de efeito de ambiente permanente materno na classe de desvio-padrão i; e $e_{\mathrm{i}}=$ vetor de resíduos na classe de desvio-padrão i, da mesma dimensão de $\mathrm{y}_{\mathrm{i}}$.

Para estimação de componentes de variância e predição de valores genéticos, foi utilizado o aplicativo Multiple Trait Derivative Free Restricted Maximum Likelihood, descrito por Boldman et al. (1995). Após obtenção das estimativas de variâncias e covariâncias genéticas aditivas para todos os pesos e em cada classe de desvio-padrão fenotípico, foram calculadas as eficiências das respostas esperadas à seleção no nível de baixa produção pela seleção no nível de alta produção, em relação à resposta direta, conforme apresentado por Araújo et al. (2009).

Do total de 211 reprodutores com proles com registros de produção, 128, 129 e 135 possuíam proles em ambas as classes de desvios-padrão para os pesos aos 365, 450 e 550 dias de idade, respectivamente (Tabela 1).

De posse dos valores genéticos dos reprodutores com proles em ambas as classes de desvio-padrão, selecionaram-se amostras contendo 10, 20 e 30\% dos melhores reprodutores, classificados em cada peso pelos valores genéticos obtidos em análise geral. Nessas amostras aplicou-se a correlação de Spearmam entre o mesmo peso, para avaliar a consistência da ordenação dos valores genéticos dos animais, utilizando os componentes de variância obtidos pelos dois processos de estimação de forma a caracterizar a influência de variâncias heterogêneas sobre o ordenamento correto dos animais, bem como a influência da intensidade de seleção aplicada.

Tabela 1 - Número de reprodutores e número médio de proles por reprodutor em cada classe de desvio-padrão para cada

\begin{tabular}{|c|c|c|c|}
\hline \multicolumn{4}{|c|}{ Peso aos 365 dias } \\
\hline Análise & Reprodutores & Proles & por reprodutor \\
\hline Geral & 211 & & 57,47 \\
\hline Alta & 175 & & 31,45 \\
\hline Baixa & 164 & & 40,38 \\
\hline \multicolumn{4}{|c|}{ Peso aos 450 dias } \\
\hline Análise & Reprodutores & Proles & por reprodutor \\
\hline Geral & 211 & & 57,45 \\
\hline Alta & 184 & & 36,29 \\
\hline Baixa & 156 & & 34,90 \\
\hline \multicolumn{4}{|c|}{ Peso aos 550 dias } \\
\hline Análise & Reprodutores & Proles & por reprodutor \\
\hline Geral & 211 & & 57,42 \\
\hline Alta & 180 & & 36,52 \\
\hline Baixa & 166 & & 33,39 \\
\hline
\end{tabular}
peso padronizado

\section{Resultados e Discussão}

Embora as médias estimadas em análises em que é desconsiderada a heterogeneidade de variâncias sejam intermediárias às obtidas nas análises do peso em cada classe de desvio-padrão fenotípico (Tabela 2), as estimativas de variâncias na análise geral aproximam-se mais das obtidas com o peso na classe de alto desvio-padrão fenotípico.

Enquanto as estimativas das médias dos pesos na classe de alto desvio-padrão excederam 11 a $15 \%$ os valores observados na classe de baixo desvio-padrão, as estimativas da variância fenotípica na classe de alto desvio-padrão ultrapassaram as observadas na classe de baixo em 46, 48 e $52 \%$ para os pesos aos 365, 450 e 550 dias de idade respectivamente. 
Os coeficientes de variação obtidos em todas as situações, para todos os pesos, foram próximos (14,15 a 16,57\%), indicando que, apesar da ocorrência de maior alteração nos valores das variâncias observadas na classe maior desviopadrão, houve manutenção da proporcionalidade da média em relação à variabilidade nas classes de desvios-padrão em todos os pesos.

As estimativas de herdabilidade, principalmente para os pesos padronizados aos 450 e 550 dias de idade, indicam que a raça Nelore apresenta variabilidade genética aditiva suficiente para que ocorra resposta à seleção baseada nesses pesos (Tabela 3). Por sua vez, as estimativas dos componentes de variância de ambiente permanente obtidas comprovam a importância de se considerar esse efeito no modelo e retratam que as matrizes apresentam estabilidade fenotípica no ponderal de seus produtos.
As estimativas das variâncias genética aditiva e residual observadas foram maiores na classe de alto desvio-padrão para o peso aos 365 e 450 dias de idade, porém, no peso aos 550 dias de idade, a variância aditiva foi maior na classe de menor desvio-padrão, indicando que, para o peso nessa idade, rebanhos de menor variabilidade fenotípica são também detentores de maior variabilidade genética, ou seja, a variabilidade presente na classe de alto desvio-padrão é em grande parte decorrente de fatores ambientais.

As estimativas da herdabilidade para o peso aos 365 dias nas duas classes de desvio-padrão e também na análise que se desconsiderou a heterogeneidade de variância foram próximas, 0,34 e 0,36, respectivamente. Entretanto, mesmo sob valores similares de herdabilidade, a correlação genética de 0,71 obtida para esse peso nas classes de alto e de baixo desvio-padrão fenotípico indica presença de interação

Tabela 2 - Número de observações e de rebanhos, médias, variâncias e coeficiente de variação para os pesos aos 365 , 450 e 550 dias de idade desconsiderando a heterogeneidade de variância geral nas classes desvio-padrão alto e baixo

\begin{tabular}{|c|c|c|c|c|c|}
\hline Análise & Observações & Rebanhos & Média & Variância & $\mathrm{CV}$ \\
\hline \multicolumn{6}{|c|}{ Peso aos 365 dias } \\
\hline Geral & 12.127 & 34 & 234,38 & $1.456,94$ & 16,29 \\
\hline Alto & 5.504 & 15 & 253,32 & $1.404,01$ & 14,79 \\
\hline Baixo & 6.623 & 19 & 218,65 & 956,66 & 14,15 \\
\hline \multicolumn{6}{|c|}{ Peso aos 450 dias } \\
\hline Geral & 12.122 & 34 & 276,15 & $2.093,97$ & 16,57 \\
\hline Alto & 6.677 & 17 & 291,97 & $2.093,98$ & 15,67 \\
\hline Baixo & 5.445 & 17 & 256,76 & $1.412,25$ & 14,64 \\
\hline \multicolumn{6}{|c|}{ Peso aos 550 dias } \\
\hline Geral & 12.117 & 34 & 320,53 & $2.744,71$ & 16,35 \\
\hline Alto & 6.573 & 16 & 335,84 & $2.925,72$ & 16,09 \\
\hline Baixo & 5.544 & 18 & 302,38 & $1.929,84$ & 14,53 \\
\hline
\end{tabular}

CV = Coeficiente de variação.

Tabelas 3 - Estimativas de componentes de variâncias, de herdabilidade e de repetibilidade para os pesos aos 365, 450 e 550 dias de idade desconsiderando a heterogeneidade de variância geral e nas classes de desvio-padrão alto e baixo

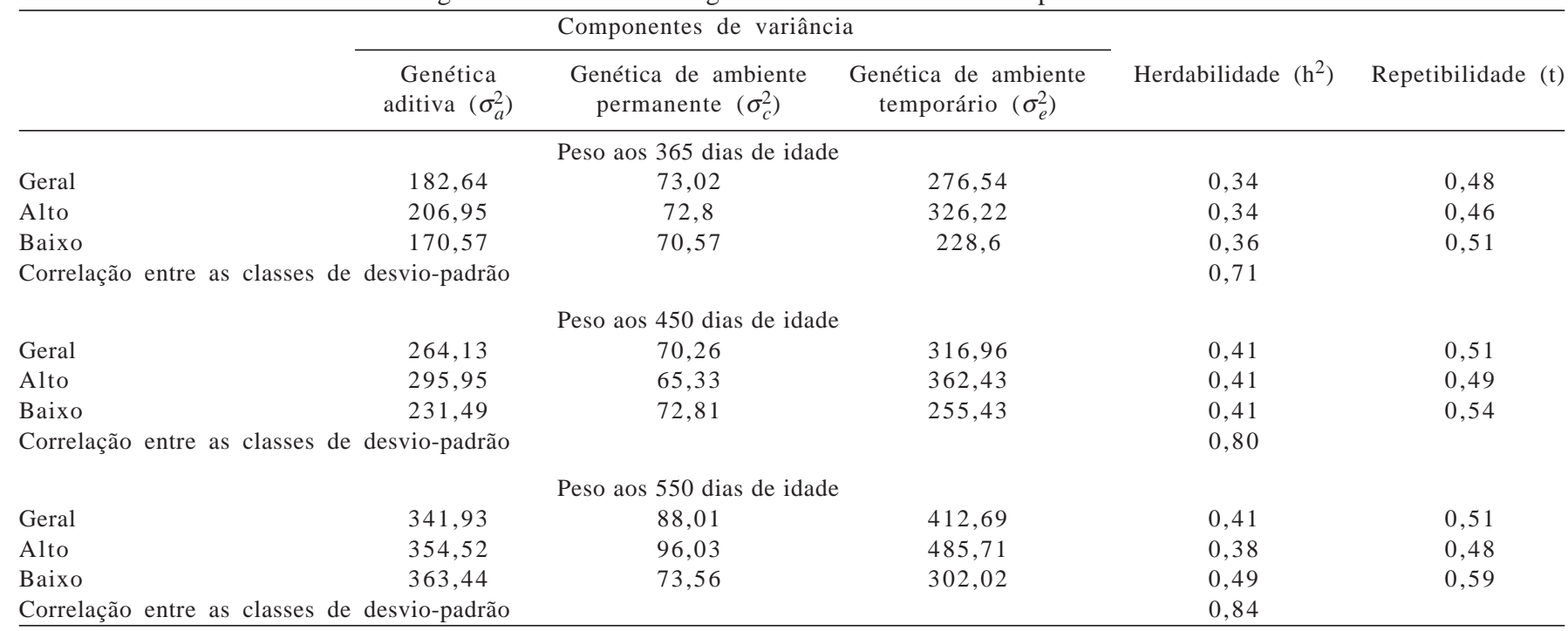


genótipo $\times$ ambiente, tendo em vista afirmações de Robertson (1959) de que valor da correlação genética menor que 0,8 significa que a interação genótipo $\times$ ambiente passa a assumir papel importante na expressão da característica. Assim, esperava-se que ocorressem alterações no ordenamento dos reprodutores, tendo em vista a heterogeneidade de variância nos dados para essa idade na raça avaliada.

Para o peso aos 450 dias, houve aumento de $27 \%$ da variância genética aditiva, decréscimo de $11 \%$ da variância de ambiente permanente e, ainda, acréscimo de $42 \%$ da variância de ambiente temporário na classe de alto desviopadrão em comparação às estimativas obtidas na classe de baixo desvio-padrão fenotípico. Novamente, a estimativa de herdabilidade, considerando ou não a heterogeneidade de variâncias, não se alterou, porém, a correlação genética entre o mesmo peso nas duas classes de desvio-padrão foi de 0,80 e isso indica redução da heterogeneidade de variâncias e da importância da interação genótipo × ambiente nessa idade.

No peso aos 550 dias de idade, a variância genética aditiva foi menor na classe de alto desvio-padrão, resultando em menor estimativa de herdabilidade quando comparada à estimativa obtida na classe de baixo desvio-padrão, visto que o mesmo não ocorreu com a variância residual. Todavia, o valor da estimativa de herdabilidade foi próximo ao da análise em que se desconsiderou a heterogeneidade de variâncias.

A maior estimativa de herdabilidade presente na classe de baixo desvio-padrão fenotípico (comparada à estimativa obtida na classe de alto desvio-padrão) decorrente da maior variabilidade genética aditiva nessa classe e, ainda, maior variabilidade ambiental na classe de alto desvio-padrão, indica que os reprodutores seriam classificados para o peso aos 550 dias em função do melhor ambiente, uma vez que reprodutores com maior número de proles em rebanhos detentores de alta variabilidade fenotípica, teriam sua predição de valor genético superestimada, em relação aos reprodutores com maior número de proles em rebanhos de menor variabilidade fenotípica.

A correlação genética entre as classes de desviopadrão foi igual a 0,84 , indicando que a variância genética da característica nas duas classes de desvio-padrão, oscilou de forma similar, implicando com isso em pequena alteração na classificação dos mesmos reprodutores nas duas classes de desvio-padrão.

Assim, o resultado obtido nesse banco de dados está de acordo com pesquisas na literatura que trataram esse assunto de forma similar em bovinos. Campelo et al. (2003) constataram valores de componentes de variâncias genética e residual, aumentando da classe de baixo para a de alto desvio-padrão fenotípico, para pesos padronizados aos 120, 240 e 420 dias de idade na raça Tabapuã e atribuíram esse comportamento à interação genótipo ambiente. Toral et al. (2004) analisaram pesos do nascimento aos 18 meses de idade em animais da raça Nelore, estratificando os dados por microrregiões do estado de Mato Grosso do Sul, e verificaram que os componentes de variância aditivos direto e residuais e as estimativas de herdabilidade foram diferentes entre as regiões, evidenciando a presença de interação genótipo $\times$ região. Com metodologia similar, Fridrich et al. (2008) verificaram em animais da raça Canchim presença de interação genótipo $\times$ unidades federativas (estados) no peso ao ano de idade, que foi mais marcante nas combinações "Região Sul com Centro Oeste" e "Sul com Norte". Com base nesse resultado, os autores sugeriam que a avaliação genética de bovinos deveria ser regional quando envolver regiões com condições ambientais bastante distintas.

Outro procedimento que comprova a influência da heterogeneidade de variâncias na avaliação genética de reprodutores utilizado nessa pesquisa foi recorrer ao cálculo da eficiência da resposta correlacionada, com a seleção dos melhores animais, feita pela classificação com base nos componentes de variâncias e covariâncias genética obtidos na classe de alto desvio-padrão fenotípico, porém, com a mesma intensidade de seleção em ambas as classes. Os resultados obtidos confirmaram eficiência de apenas 69 e,80 e 73\% para os pesos padronizados aos 365, 450 e 550 dias de idade, respectivamente. Portanto, a identificação dos melhores reprodutores com base no desempenho de sua prole em ambiente alta variabilidade fenotípica obrigatoriamente pode não implicar em melhores reprodutores para rebanhos nos quais essa variabilidade é baixa.

Para os pesos aos 365 e 450 dias de idade, as estimativas de repetibilidade foram próximas em todas as situações. Entretanto, aos 550 dias de idade, houve incremento de 60 e 30\% nas estimativas de variância de ambiente temporário e de ambiente permanente, respectivamente, na classe de alto desvio-padrão em relação à classe de baixo desviopadrão fenotípico, resultando em maior estimativa de repetibilidade nessa última classe, como resultado da heterogeneidade de variância de origem ambiental.

O interesse dos criadores de bovinos é que os animais de reprodução sejam avaliados e ordenados corretamente, independentemente do critério de avaliação genética, de forma a maximizar o progresso genético no rebanho, bem como os lucros da atividade, como afirmaram Van Melis et al. (2003). Nesta pesquisa utilizou-se a correlação de Spearman para avaliar a consistência da ordenação dos animais com base nos seus valores genéticos, estimados com os 
Tabela 4 - Correlação de Spearman para os valores genéticos de reprodutores para os pesos padronizados aos 365 , 450 e 550 dias de idade em análise geral e em cada classe de desvio-padrão fenotípico

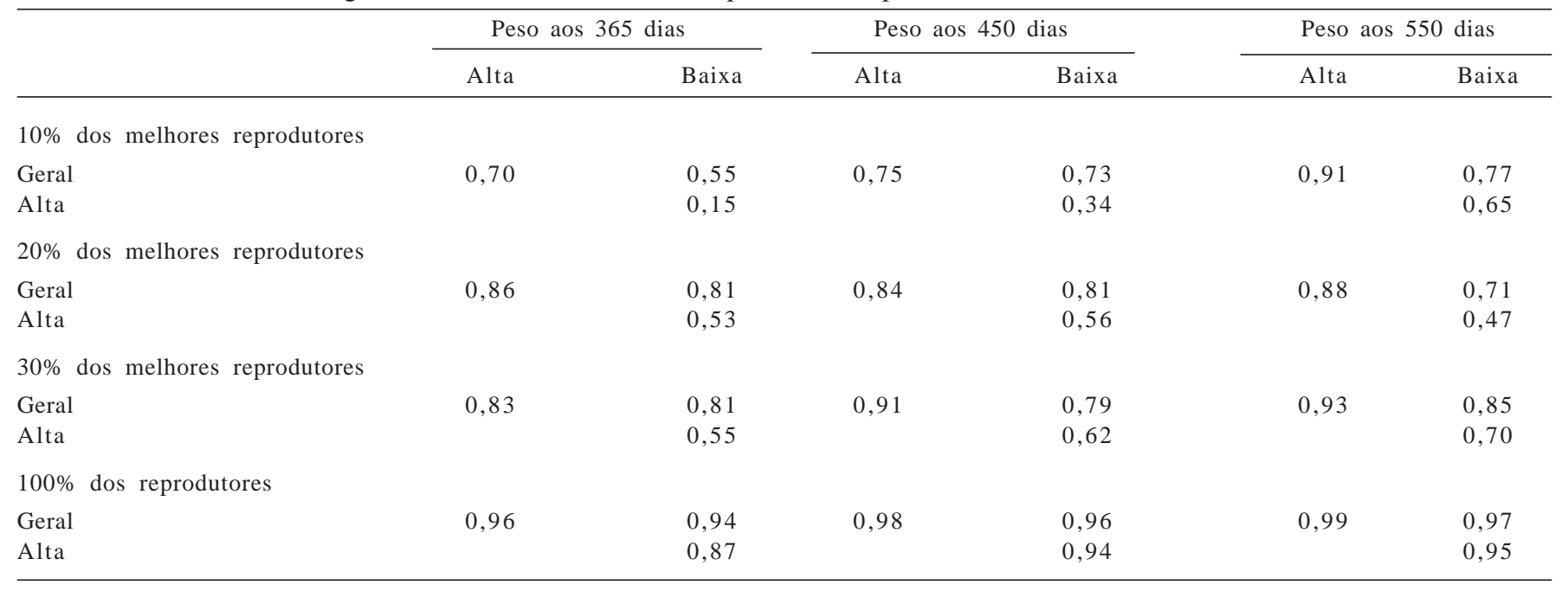

componentes de variância obtidos nos dois critérios testados, que são "considerando" e "não considerando" a heterogeneidade de variâncias dos dados (Tabela 4).

Foi marcante a influência da pressão de seleção sobre o ordenamento dos reprodutores quando se considerou a heterogeneidade de variância fenotípica. Na ausência de pressão de seleção, a correlação de ordem entre valores genéticos dos reprodutores com prole em ambas as classes de desvio-padrão nas três combinações possíveis foi superior a 0,87, indicando que não há alteração relevante no ordenamento em cada peso. Todavia, quanto maior foi a intensidade de seleção considerada, menor a coincidência entre ordenamento dos reprodutores com base nos valores genéticos preditos nas classes de desvio-padrão e também entre elas e a análise geral.

Destaca-se que sob maior intensidade de seleção (escolha de 10\% dos melhores animais), a correlação entre os valores genéticos obtidos na classe de baixo desviopadrão com aqueles obtidos nas demais situações foi a mais baixo, indicando que a interação genótipo $\times$ ambiente se apresenta de forma mais marcante para os animais de maior valor genético.

Resultado similar foi verificado por Araújo et al. (2009) em bovinos leiteiros. Esses autores verificaram menores valores de correlações de Spearman em maiores intensidades seletivas nos reprodutores. Campelo et al. (2003), considerando a amostra com todos os reprodutores avaliados, verificaram altas correlações de Spearman para os valores genéticos preditos dos reprodutores entre as classes de desvios-padrão, como também foi verificado neste estudo.
Embora amostras menores sejam mais sensíveis a pequenas alterações no ordenamento dos reprodutores, neste estudo essas alterações tiveram grande influência no valor econômico de comercialização do sêmen, principalmente na alteração do ordenamento das primeiras colocações. Desse modo, o maior impacto da presença da heterogeneidade de variância sobre a avaliação genética dos reprodutores reside sobre a classificação dos animais considerados elites.

Para o peso padronizado aos 550 dias, em que a herdabilidade na classe de baixo desvio-padrão foi maior que nas duas outras análises, mesmo assim a correlação de ordem foi baixa, logo, a presença de heterogeneidade de variância pode ter sido decorrente de fatores não-genéticos. Assim, a seleção sem considerar a heterogeneidade de variâncias poderia ser mais em função do melhor ambiente, e não do melhor genótipo.

\section{Conclusões}

Há interação genótipo $\times$ ambiente sob a forma de heterogeneidade de variâncias, com variâncias maiores em rebanhos mais variáveis. O impacto dessa interação é mais prejudicial para peso padronizado aos 550 dias de idade, visto que a origem da heterogeneidade de variâncias é decorrente de fatores de ambiente. A presença de heterogeneidade de variâncias causa maior impacto sobre a avaliação genética dos reprodutores sob intensidade de seleção elevada, sendo interessante sua consideração em programas de avaliação genética desta raça, a fim de se obterem progressos genéticos, via seleção, de forma mais precisa. 


\section{Referências}

ARAUJO, C.V.; RESENDE, G.S.S.; ARAÚJO, S.I. et al. Interação genótipo $\mathrm{x}$ ambiente para produção de leite na raça Pardo Suíço, utilizando-se inferência bayesiana. Acta Scientiarum Animal Science, v.3, n.2, p.205-211, 2009.

BOLDMAN, K.G.; KRIESE, L.A.; VAN VLECK, L.D. et al. A manual for use of MTDFREML: a set of programs to obtain estimates of variance and covariance. Lincoln: Agricultural Research Service, 1995. 120p.

CAMPELO, J.E.G.; LOPES, P.S.; TORRES, R.A. et al. Influência da heterogeneidade de variâncias na avaliação genética de bovinos de corte da raça Tabapuã, Arquivo Brasileiro de Medicina Veterinária e Zootecnia, v.55, n.6, p.685-693, 2003.

CARNEIRO, A.P.S.; TORRES, R.A.; LOPES, P.S. et al. Erros na classificação de touros, vacas e touros jovens geneticamente superiores avaliados na presença de heterogeneidade, Revista Brasileira de Zootecnia, v.35, n.5, p.1907-1914, 2006.
FRIDRICH, A.B.; SILVA, M.A.; VALENTE, B.D. et al. Interação genótipo $\mathrm{x}$ ambiente e estimativas de parâmetros genéticos dos pesos aos 205 e 365 dias de idade de bovinos Nelore. Arquivo Brasileiro de Medicina Veterinária e Zootecnia, v.60, n.4, p.785-793, 2008.

MARTINS, E.N. Avaliação genética e heterogeneidade de variância. In: REUNIÃO ANUAL DA SOCIEDADE BRASILEIRA DE ZOOTECNIA, 39., 2002, Recife. Anais... Sociedade Brasileira de Zootecnia, 2002. (CD-ROM).

ROBERTSON, A. The sampling variance of the genetic correlation coefficient. Biometrics, v.15, p.469-485, 1959.

TORAL, F.L.B.; SILVA, L.O.C.; MARTINS, E.N. et al. Interação genótipo $\mathrm{x}$ ambiente em características de crescimento de bovinos da raça Nelore no Mato Grosso do Sul. Revista Brasileira de Zootecnia, v.33, n.6, p.1445-1455, 2004.

VAN MELIS, M.H.; ELER, J.P.; SILVA, J.A.V. et al. Estimação de Parâmetros Genéticos em Bovinos de Corte Utilizando os Métodos de Máxima Verossimilhança Restrita e R. Revista Brasileira de Zootecnia, v.32, n.6, p.1624-1632, 2003 (supl. 1). 